\title{
EXTENSION OF CONTINUOUS FUNCTIONS INTO UNIFORM SPACES
}

\author{
SALVADOR HERNÁNDEZ
}

\begin{abstract}
Let $X$ be a dense subspace of a topological space $T$, let $Y$ be a uniformizable space, and let $f: X \rightarrow Y$ a continuous map. In this paper we study the problem of the existence of a continuous extension of $f$ to the space $T$. Thus we generalize basic results of Taimanov, Engelking and BlefkoMrówka on extension of continuous functions. As a consequence, if $D$ is a nest generated intersection ring on $X$, we obtain a necessary and sufficient condition for the existence of a continuous extension to $v(X, D)$, of a continuous function over $X$, when the range of the map is a uniformizable space, and we apply this to realcompact spaces. Finally, we suppose each point of $T \backslash X$ has a countable neighbourhood base, and we obtain a generalization of a theorem by Blair, herewith giving a solution to a question proposed by Blair.
\end{abstract}

In the sequel the word space will designate a topological space. By $\mu X$ we shall denote a uniform space, $\mu$ being a collection of covers in the sense of Tukey $[\mathbf{1 3}]$ and Isbell [10]. $\gamma \mu X$ will mean the completion of the space $\mu X$. Let $\mu$ be a uniformity on $X$ and let $m$ be an infinite'cardinal number, then $\mu_{m}$ will denote the uniformity on $X$.generated by the $\mu$-covers of power $<m$ (we only consider $\mu$ and $m$ for which $\mu_{m}$ is definable; see [6,10 and 14]). The compact uniform space $\gamma \mu_{\aleph_{0}} X$ is called the Samuel compactification of $\mu X$, denoted by $s \mu X$. By $\tau \mu X$ we shall denote the set $X$ equipped with the $\mu$-uniform topology. We say that $E$ and $D$ subsets of $X$ are $\mu$-separated when $E$ is far from $D$ in the proximity defined by $\mu$.

By a base on a space $X$ we mean a nest generated intersection ring (or equivalently, a strong delta normal base) in $X[\mathbf{1}$ and 10]. It is known that each base $D$ on $X$ has associated a Wallman compactification $W(X, D)$ and a Wallman realcompactification $v(X, D)$. If $\mu X$ is a uniform space, we shall denote by $Z(\mu X)$ the base on $\tau \mu X$ formed by uniform zero-sets (see [7 and 8]), $v(\mu X)$ will denote the space $v(X, Z(\mu X))$ and $\beta(\mu X)$ the space $W(X, Z(\mu X))$.

Let $T$ be a space and suppose $X \subseteq T$. If $m$ is a cardinal number, we say that $X$ is $m$-dense in $T$ when for every family $\left\{U_{i}: i \in I\right\}$, with $|I|<m$, of nonvoid open sets in $T$ such that $\bigcap\left\{U_{i}: i \in I\right\} \neq \varnothing$, it follows that $\bigcap\left\{U_{i} \cap X: i \in I\right\} \neq \varnothing$. If $A$ is a subset of $T$, we define the $m$-closure of $A$ in $T$ as the union of all subspaces of $T$ in which $A$ is $m$-dense.

The following result generalizes the Taimanov theorem [12] of extension of continuous functions into compact spaces.

THEOREM 1. Let $T$ be a space in which $X$ is $m$-dense, let $\mu Y$ be a uniform space, and let $f: X \rightarrow \tau \mu Y$ be a continuous map. Then the following are equivalent:

(a) $f$ has a continuous extension $\bar{f}: T \rightarrow \tau \gamma \mu_{m} Y$.

Received by the editors December 14, 1984 and, in revised form, June 28, 1985.

1980 Mathematics Subject Classification. Primary 54C20, 54C30, 54D60. 
(b) For every pair $E$ and $D$ of subsets of $Y$ which are $\mu$-separated, we have that $\operatorname{cl}_{T} f^{-1}(E) \cap \operatorname{cl}_{T} f^{-1}(D)=\varnothing$, i.e., $f^{-1}(E)$ and $f^{-1}(D)$ have disjoint closures in $T$.

ProOF. $(\mathrm{a}) \Rightarrow(\mathrm{b})$. This is clear.

(b) $\Rightarrow$ (a). Let $p \in T \backslash X$ and let $\mathcal{F}_{p}$ be the trace on $X$ of the neighbourhood filter of $p$. In order to prove that there is a continuous extension of $f$, it is sufficient with showing that the filter generated by $f\left(\mathcal{F}_{p}\right)=\left\{f(F): F \in \mathcal{F}_{p}\right\}$ is a Cauchy filter in $\mu_{m} Y$. Let $\mathcal{U} \in \mu_{m}$ arbitrary and consider $\mathcal{V} \in \mu_{m}$ a star-refinement of $\mathcal{U}$. Since $|\mathcal{V}|<m$ and $X$ is $m$-dense in $T$, there is $V_{0} \in \mathcal{V}$ with $f(F) \cap V_{0} \neq \varnothing$ for every $F \in \mathcal{F}_{p}$. If not, let $\mathcal{V}=\left\{V_{i}: i \in I\right\}$ with $|I|<m$; for every $i \in I$ there is $F_{i} \in \mathcal{F}_{p}$ such that $f\left(F_{i}\right) \cap V_{i}=\varnothing$. As $\bigcup\left\{V_{i}: i \in I\right\}=Y$, it follows that $\bigcap\left\{f\left(F_{i}\right): i \in I\right\}=\varnothing$ and, consequently, $\bigcap\left\{F_{i}: i \in I\right\}=\varnothing$, which is a contradiction.

Now let us see that there is $F_{0} \in \mathcal{F}_{p}$ such that $f\left(F_{0}\right) \subseteq \operatorname{st}\left(V_{0}, \mathcal{V}\right)$, i.e., $f\left(F_{0}\right)$ is contained in the star of $V_{0}$ with respect to $\mathcal{V}$.

Let us suppose that, for every $F \in \mathcal{F}_{p}, f(F) \cap\left(Y \backslash \operatorname{st}\left(V_{0}, \mathcal{V}\right)\right) \neq \varnothing$. Then we can define the following two sets: $A=\bigcup\left\{f(F) \cap V_{0}: F \in \mathcal{F}_{p}\right\}$ and $B=$ $\bigcup\left\{f(F) \backslash \operatorname{st}\left(V_{0}, \mathcal{V}\right): F \in \mathcal{F}_{p}\right\}$. We have that $A \subseteq V_{0}$ and $B \cap \operatorname{st}\left(V_{0}, \mathcal{V}\right)=\varnothing$. This shows that $A$ and $B$ are $\mu$-separated. However, $p \in \operatorname{cl}_{T} f^{-1}(A) \cap \operatorname{cl}_{T} f^{-1}(B)$, which is a contradiction.

Thus there exists $F_{0} \in \mathcal{F}_{p}$ with $f\left(F_{0}\right) \subseteq \operatorname{st}\left(V_{0}, \mathcal{V}\right)$. Since $\mathcal{V}$ is a star-refinement of $\mathcal{U}$, there is $U_{0} \in \mathcal{U}$ such that $f\left(F_{0}\right) \subseteq U_{0}$. This proves that the filter generated by $f\left(\mathcal{F}_{p}\right)$ is a Cauchy filter in $\mu_{m} Y$.

COROLlaRY 2. Let $\mu X$ be a uniform space. Then $\tau \gamma \mu_{m} X$ contains the $m$ closure of $\tau \mu X$ in $\tau s \mu X$.

ProOF. Let $T$ be the $m$-closure of $\tau \mu X$ in $\tau s \mu X$, and consider $f: \tau \mu X \rightarrow$ $\tau \mu X$ the identity map. By applying Theorem 1 there is a continuous extension $\bar{f}: T \rightarrow \tau \gamma \mu_{m} X \subseteq \tau s \mu X$. Let $g: \tau s \mu X \rightarrow \tau s \mu X$ be the identity map and consider $g_{\left.\right|_{T}}: T \rightarrow \tau s \mu X$. As $\bar{f}$ and $g_{\left.\right|_{T}}$ coincide in a dense subspace of $T$, it follows that $\bar{f}=g_{\left.\right|_{T}}$. Therefore, $\bar{f}$ is the identity map and $T \subseteq \tau \gamma \mu_{m} X$.

COROLlaRY 3. Let $\mu_{1}$ and $\mu_{2}$ be two separable (see [8]) uniformities on a space $X$ and suppose that $\tau \mu_{i} X$ is $Q$-dense ( $\aleph_{1}$-dense) in $\tau \gamma \mu_{i} X$, for $i=1,2$. Then $\tau \gamma \mu_{1} X=\tau \gamma \mu_{2} X$ if and only if for every pair $E$ and $D$ of subsets of $X$ which are $\mu_{i}$-separated, $\operatorname{cl}_{\tau \gamma \mu_{j} X} E \cap \operatorname{cl}_{\tau \gamma \mu_{j} X} D=\varnothing$ for $i \neq j, 1 \leq i, j \leq 2$.

PROOF. The proof is clear by taking $f: \tau \mu_{i} X \rightarrow \tau \mu_{j} X$ as the identity map in Theorem 1 , for $i \neq j, 1 \leq i, j \leq 2$.

The following result generalizes [5, Theorem 2 and $\mathbf{3}$, Theorem $\mathrm{C}]$.

THEOREM 4. Let $T$ be a space in which $X$ is dense, let $\mu Y$ be a uniform space, and let $f: X \rightarrow \tau \mu Y$ be a continuous map. Then $f$ has a continuous extension $\bar{f}: T \rightarrow \tau \gamma \mu_{m} Y$ if and only if

(a) For every pair $E$ and $D$ of subsets of $Y$ which are $\mu$-separated, we have that $\mathrm{cl}_{T} f^{-1}(E) \cap \mathrm{cl}_{T} f^{-1}(D)=\varnothing$.

(b) If $\left\{F_{i}: i \in I\right\},|I|<m$, is a family of subsets of $Y$ which has the finite intersection property and $\bigcap\left\{\operatorname{cl}_{\tau \gamma \mu_{m} Y} F_{i}: i \in I\right\}=\varnothing$, then $\bigcap\left\{\operatorname{cl}_{T} f^{-1}\left(F_{i}\right): i \in I\right\}=$ $\varnothing$.

PROOF. The necessity is clear. 
Sufficiency. Consider $f X \rightarrow \tau \mu Y$. By condition (a) we can apply Theorem 1 for $m=\aleph_{0}$. So there is a continuous extension $\bar{f}: T-\tau s \mu Y$. Now, suppose there is $p \in$ $T \backslash X$ such that $\bar{f}(p) \notin \tau \gamma \mu_{m} Y$. By Corollary 2, applied to $\tau \gamma \mu_{m} Y$, we have that the $m$-closure of $\tau \gamma \mu_{m} Y$ in $\tau s \mu Y$ is $\tau \gamma \mu_{m} Y$. Hence there is a family $\left\{V_{i}: i \in I\right\},|I|<m$, of closed neighbourhoods of $\bar{f}(p)$ in $\tau s \mu Y$ such that $\bigcap\left\{V_{i} \cap\left(\tau \gamma \mu_{m} Y\right): i \in I\right\}=\varnothing$. Consider the family of sets $\left\{V_{i} \cap(\tau \mu Y): i \in I\right\}$. This family verifies the hypotheses of (b). However, $p \in \bigcap\left\{\operatorname{cl}_{T} f^{-1}\left(V_{i} \cap(\tau \mu Y)\right): i \in I\right\}$ which is a contradiction. This proves that $\bar{f}(T) \subseteq \tau \gamma \mu_{m} Y$.

From Theorem 1 the following result on spaces with bases is derived.

THEOREM 5. Let $\mu X$ and $\nu Y$ be uniform spaces and let $f: \tau \mu X \rightarrow \tau \nu Y$ be $a$ continuous map. Then the following are equivalent:

(a) There is a continuous ext. $\bar{f}: v(\mu X) \rightarrow \tau s \nu Y$.

(b) There is a continuous ext. $\bar{f}: v(\mu X) \rightarrow \beta(\nu Y)$.

(c) There is a continuous ext. $\bar{f}: v(\mu X) \rightarrow v(\nu Y)$.

(d) If $D$ and $E$ are $\nu$-separated in $Y$, then

$$
\operatorname{cl}_{v(\mu X)} f^{-1}(D) \cap \operatorname{cl}_{v(\mu X)} f^{-1}(E)=\varnothing .
$$

(e) If $D$ and $E$ are $Z(\nu Y)$-separated, then

$$
\operatorname{cl}_{v(\mu X)} f^{-1}(D) \cap \operatorname{cl}_{v(\mu X)} f^{-1}(E)=\varnothing .
$$

(f) If $D$ and $E$ are disjoint in $Z(\nu Y)$, then

$$
\operatorname{cl}_{v(\mu X)} f^{-1}(D) \cap \operatorname{cl}_{v(\mu X)} f^{-1}(E)=\varnothing .
$$

PROOF. (a) $\Leftrightarrow(d)$. This is Theorem 1 .

(c) $\Leftrightarrow(e)$. This is a consequence of Theorem 1, by considering in $Y$ the uniformity associated to the base $Z(\nu Y)$ (see $[7,6.5(\mathrm{a})]$ ).

(c) $\Rightarrow(\mathrm{b}),(\mathrm{b}) \Rightarrow(\mathrm{f})$ and $(\mathrm{f}) \Rightarrow(\mathrm{e})$. These are clear.

(c) $\Rightarrow$ (a). By [7, Theorem 4.2] $v(\nu Y)$ is the $Q$-closure of $\tau \nu Y$ in $\tau s \nu Y$.

(a) $\Rightarrow(\mathrm{c})$. As $\tau \mu X$ is $Q$-dense in $v(\mu X)$, it follows that $\bar{f}(v(\mu X)) \subseteq v(\nu Y)$.

Let $X$ be a completely regular Hausdorff space. We denote by $X^{\prime}$ the set $X$ endowed with the $P$-topology associated, i.e., the topology for which the collection of $G_{\delta}$-subsets of $X$ forms an open base. If $D$ is a base on $X$, then by $\sigma(D)$ we mean the $\sigma$-algebra of sets generated by $D$. It is clear that $\sigma(D)$ is a base on $X^{\prime}$.

The next proposition is an application to realcompact spaces of the results above.

PROPOSITION 6. Let $X$ be a realcompact space. If $\tilde{X}$ is the set $X$ endowed with a topology such that $\tilde{X}^{\prime}=X^{\prime}$ and, for every pair $E$ and $D$ of disjoint Baire sets of $X$, we have that

$$
\operatorname{cl}_{v\left(X^{\prime}, \sigma(Z(\tilde{X}))\right)} E \cap \mathrm{cl}_{v\left(X^{\prime}, \sigma(Z(\tilde{X}))\right)} D=\varnothing,
$$

then $\tilde{X}$ is realcompact.

Proof. Let $f: \tilde{X}^{\prime} \rightarrow X^{\prime}$ be the identity map. By Theorem 5 , there is a continuous extension $\bar{f}: v(\tilde{X} ; \sigma(Z(\tilde{X}))) \rightarrow v\left(X^{\prime}, \sigma(Z(X))\right)$. Now, by [9, Theorem 16], $v\left(X^{\prime}, \sigma(Z(X))\right)=X^{\prime}$. Then $\bar{f}\left(v\left(\tilde{X}^{\prime}, \sigma(Z(\tilde{X}))\right)\right)=X^{\prime}$. Since $f$ is a homeomorphism, it follows that $v\left(\tilde{X}^{\prime}, \sigma(Z(\tilde{X}))\right)=\tilde{X}^{\prime}$. Therefore $\tilde{X}$ is realcompact, as a consequence of $[9$, Theorem 16$]$.

We are informed by the referee that the following result has also been proved by Comfort and Retta in [4]. 
COROLlaRY 7. Let $X$ be a realcompact space, and let $\tilde{X}$ be the set $X$ endowed with a topology finer than the topology of $X$ and such that $\tilde{X}^{\prime}=X^{\prime}$. Then $\tilde{X}$ is also realcompact.

ProOF. Since $\sigma(Z(\tilde{X})) \supseteq \sigma(Z(X))$ we can apply Proposition 6 .

In [2] Blair proved the following result.

THEOREM 8. Let $X$ be a dense subspace of a topological space $T$, assume each $p \in T \backslash X$ has a countable base of neighbourhoods, let $Y$ be a closed subspace of $R$, and let $f: X \rightarrow Y$ be continuous. Then the following are equivalent:

(a) $f$ extends continuously over $T$.

(b) If $F_{1}$ and $F_{2}$ are disjoint countable closed subsets of $Y$, then $f^{-1}\left(F_{1}\right)$ and $f^{-1}\left(F_{2}\right)$ have disjoint closures in $T$.

Also Blair proposed in [2] the question of possible generalizations of Theorem 8 for Tychonoff spaces $Y$ that are not necessarily closed subspaces of $R$. What follows is a solution to this question.

THEOREM 9. Let $X$ be a dense subspace of $T$, assume each point $p \in T \backslash X$ has a countable base of neighbourhoods, let $\mu Y$ be a uniform space, and let $f: X \rightarrow \tau \mu Y$ be a continuous map. Then the following are equivalent:

(a) $f$ has a continuous extension $\vec{f}: T \rightarrow \tau \gamma \mu Y$.

(b) If $F_{1}$ and $F_{2}$ are countable subsets of $Y$ which are $\mu$-separated, then

$$
\operatorname{cl}_{T} f^{-1}\left(F_{1}\right) \cap \operatorname{cl}_{T} f^{-1}\left(F_{2}\right)=\varnothing .
$$

ProOF. $(a) \Rightarrow(b)$. This is clear.

(b) $\Rightarrow($ a). First we shall prove that if $E$ and $D$ are two arbitrary subsets of $Y$ which are $\mu$-separated, then $\operatorname{cl}_{T} f^{-1}(E) \cap \mathrm{cl}_{T} f^{-1}(D)=\varnothing$. Let us suppose there is $p \in \operatorname{cl}_{T} f^{-1}(E) \cap \operatorname{cl}_{T} f^{-1}(D)$. Then there is $\left\{x_{n}: n \in N\right\} \subseteq f^{-1}(E)$ and $\left\{y_{n}: n \in N\right\} \subseteq f^{-1}(D)$ such that $\lim x_{n}=\lim y_{n}=p$. Let $F_{1}=\left\{f\left(x_{n}\right)\right\}$ and $F_{2}=\left\{f\left(y_{n}\right)\right\}$. Then $F_{1}$ and $F_{2}$ are $\mu$-separated, but $p \in \operatorname{cl}_{T} f^{-1}\left(F_{1}\right) \cap \operatorname{cl}_{T} f^{-1}\left(F_{2}\right)$, which is a contradiction.

Thus, by Theorem 1 , there is a continuous extension $\bar{f}: T \rightarrow \tau s \mu Y$. Suppose there is $p \in T \backslash X$ with $\bar{f}(p) \in \tau s \mu Y \backslash \tau \gamma \mu Y$. Let us consider $\left\{x_{n}: n \in N\right\} \subseteq X$ with $\lim x_{n}=p$ and define $A_{m}=\left\{f\left(x_{n}\right): n \geq m\right\}$. Since $\bar{f}(p) \notin \tau \gamma \mu Y$, there is a $\mu$-cover $\mathcal{U}$ such that $A_{m} \cap(Y \backslash \operatorname{st}(U, \mathcal{U})) \neq \varnothing$ for all $m \in N$ and $U \in \mathcal{U}$. Take $x_{1}$. Then there is $m_{1} \in N$ such that $\operatorname{st}\left(\left\{f\left(x_{1}\right)\right\}, \mathcal{U}\right) \cap A_{m}=\varnothing$ for all $m \geq m_{1}$. If not, we define $A=A_{1} \cap \operatorname{st}\left(\left\{f\left(x_{1}\right)\right\}, \mathcal{U}\right)$ and $B=A_{1} \cap(Y \backslash \operatorname{st}(U, \mathcal{U}))$, where $U \in \mathcal{U}$ and $f\left(x_{1}\right) \in U$. We have that $A$ and $B$ are $\mu$-separated but $p \in \operatorname{cl}_{T} f^{-1}(A) \cap \operatorname{cl}_{T} f^{-1}(B)$, which is a contradiction.

Consider now a subsequence of $\left\{x_{n}\right\}$ defined as follows: Let $y_{1}=x_{1}$. Then there is $m_{1} \in N$ such that $\operatorname{st}\left(\left\{f\left(x_{1}\right)\right\}, \mathcal{U}\right) \cap A_{m}=\varnothing$ if $m \geq m_{1}$. Let $y_{2}=x_{m_{1}}$. In the same way there is $m_{2} \in N$ such that $\operatorname{st}\left(\left\{f\left(y_{2}\right)\right\}, \mathcal{U}\right) \cap A_{m}=\varnothing$ if $m \geq m_{2}$. Let $y_{3}=x_{m_{2}}, \ldots$. Thus we have defined a subsequence $\left\{y_{n}\right\}$ of $\left\{x_{n}\right\}$ such that if $i \neq j$ then $f\left(y_{j}\right) \notin \operatorname{st}\left(\left\{f\left(y_{i}\right)\right\}, \mathcal{U}\right)$. Let $A=\left\{f\left(y_{2 k}\right)\right\}$ and $B=\left\{f\left(y_{2 k+1}\right)\right\}$. Then st $(A, \mathcal{U}) \cap B=\varnothing$, so $A$ and $B$ are $\mu$-separated, but $p \in \operatorname{cl}_{T} f^{-1}(A) \cap \operatorname{cl}_{T} f^{-1}(B)$. This contradiction proves that $\bar{f}(p) \in \tau \gamma \mu X$. 
COROLlARY 10. Let $X$ be a dense subspace of $T$, assume each $p \in T \backslash X$ has a countable base of neighbourhoods, let $Y$ be a Tychonoff space, and let $f: X \rightarrow Y$ be a continuous map. Then the following are equivalent:

(a) $f$ has a continuous extension $\bar{f}: T \rightarrow v Y$.

(b) If $F_{1}$ and $F_{2}$ are two countable subsets of $Y$ which are completely separated by $C(Y)$, then $\operatorname{cl}_{T} f^{-1}\left(F_{1}\right) \cap \operatorname{cl}_{T} f^{-1}\left(F_{2}\right)=\varnothing$.

ProOF. The proof follows from Theorem 9 by considering in $Y$ the weak uniformity generated by the real-valued continuous functions on $Y$.

COROLlary 11. Let $X$ be a dense subspace of $T$, assume each $p \in T \backslash X$ has a countable base of neighbourhoods, let $Y$ be a first countable Tychonoff space, and let $f: X \rightarrow Y$ be a continuous map. Then the following are equivalent:

(a) $f$ has a continuous extension $\bar{f}: T \rightarrow Y$.

(b) If $F_{1}$ and $F_{2}$ are two disjoint countable closed subsets of $Y$, then $\operatorname{cl}_{T} f^{-1}\left(F_{1}\right) \cap$ $\operatorname{cl}_{T} f^{-1}\left(F_{2}\right)=\varnothing$.

Proof. First we shall prove that if $E$ and $D$ are two subsets of $Y$ which are completely separated, then $\operatorname{cl}_{T} f^{-1}(E) \cap \mathrm{cl}_{T} f^{-1}(D)=\varnothing$. Suppose there is $p \in \operatorname{cl}_{T} f^{-1}(E) \cap \operatorname{cl}_{T} f^{-1}(D)$ and consider $\left\{x_{n}: n \in N\right\} \subseteq f^{-1}(E),\left\{y_{n}: n \in N\right\} \subseteq$ $f^{-1}(D)$ with $\lim x_{n}=\lim y_{n}=p$. We are going to define a closed subset $\bar{A}$ of $Y$ as follows: Take $A=\left\{f\left(x_{n}\right)\right\}$ if this set is closed in $Y$. If not, there is $z \in Y$ and a subsequence $\left\{f\left(x_{n_{k}}\right)\right\}$ of $\left\{f\left(x_{n}\right)\right\}$ such that $\lim f\left(x_{n_{k}}\right)=z$. In this case we take $A=\left\{f\left(x_{n_{k}}\right)\right\} \cup\{z\}$. In the same way we can define a closed subset $B$ of $Y$ from $\left\{f\left(y_{n}\right)\right\}$. Thus $A$ and $B$ are two disjoint countable closed subsets of $Y$ with $\operatorname{cl}_{T} f^{-1}(A) \cap \operatorname{cl}_{T} f^{-1}(B) \neq \varnothing$, which is a contradiction. Therefore, $\mathrm{cl}_{T} f^{-1}(E) \cap \mathrm{cl}_{T} f^{-1}(D)=\varnothing$.

By Corollary 10 there is a continuous extension $\bar{f}: T \rightarrow v Y$. Suppose there is $p \in$ $T \backslash X$ with $\bar{f}(p) \in v Y \backslash Y$. By hypothesis there is a sequence $\left\{x_{n}: n \in N\right\} \subseteq X$, with $x_{n} \neq x_{m}$ if $n \neq m$, such that $\lim x_{n}=p$. Let $A=\left\{f\left(x_{2 n}\right)\right\}$ and $B=\left\{f\left(x_{2 n+1}\right)\right\} . A$ and $B$ are disjoint countable closed subsets of $Y$ and $\operatorname{cl}_{T} f^{-1}(A) \cap \operatorname{cl}_{T} f^{-1}(B) \neq \varnothing$. This contradiction proves that $\bar{f}(T) \subseteq Y$.

EXAMPLE 12. In general, the space $v Y$ cannot be replaced by $Y$ in Corollary 10.

Let $X$ be a Tychonoff space such that there is a point $p \in v X \backslash X$ and a sequence $\left\{x_{n}\right\} \subseteq X$ with $\lim x_{n}=p$. Let $\tilde{X}$ be the space $X$ but take the point $x_{n}$ as an open set for all $n \in N$, and let $T=\tilde{X} \cup\{p\}$, where a base of neighbourhoods for $p$ is given by the sets $V_{m}=\left\{x_{n}: n \geq m\right\}, m \in N$. If we consider $f: \tilde{X} \rightarrow X$ as the identity map, then clearly $f$ verifies condition (b) of Corollary 10. Thus $f$ has a continuous extension $\bar{f}: T \rightarrow v X$ and $\bar{f}(p)=p \in v X \backslash X$.

EXAMPLE 13. The hypothesis of Corollary 11 that $Y$ be a first countable space cannot be relaxed.

Let $X=N, T=N^{*}$ and $Y=\beta N$. If we consider the identity map $f: N \rightarrow \beta N$ then, since countable closed sets in $\beta N$ are finite, we have that $f$ verifies condition (b) of Corollary 11. However, $f$ cannot be extended over $T$.

ACKNOWLEDGMENT. We thank the referee for his very valuable reflections and comments on a previous version of this paper. 


\section{REFERENCES}

1. R. A. Alo and H. L. Shapiro, Normal topological spaces, Cambridge Univ. Press, London, 1974.

2. R. L. Blair, Extension of continuous functions from dense subspaces, Proc. Amer. Math. Soc. 54 (1976), 355-359.

3. R. Blefko and S. Mrowka, On the extension of continuous functions from dense subspaces, Proc. Amer. Math. Soc. 17 (1966), 1396-1400.

4. W. W. Comfort and T. Retta, Rings of continuous functions, Lecture Notes in Pure and Appl. Math., Vol. 95, Dekker, New York, 1985.

5. R. Engelking, Remarks on real-compact spaces, Fund. Math. 55 (1964), 303-308.

6. T. E. Gantner, Extensions of uniformly continuous pseudometrics, Trans. Amer. Math. Soc. 132 (1968), 147-157.

7. A. W. Hager, On inverse-closed subalgebras of $C(X)$, Proc. London Math. Soc. (3) 19 (1969), 233-257.

8. __ Some nearly fine uniform spaces, Proc. London Math. Soc. (3) 28 (1974), 517-546.

9. E. Hewitt, Linear functionals on spaces of continuous functions, Fund. Math. 37 (1950), 161-189.

10. J. R. Isbell, Uniform spaces, Math. Surveys, No. 12, Amer. Math. Soc., Providence, R.I., 1964.

11. A. K. Steiner and E. F. Steiner, Nest generated intersection rings in Tychonoff spaces, Trans. Amer. Math. Soc. 148 (1970), 589-601.

12. A. D. Taimanov, On the extension of continuous mappings of topological spaces, Mat. Sb. 31 (1952), 459-462. (Russian)

13. J. W. Tukey, Convergence and uniformity in topology, Ann. of Math. Studies, No. 2, Princeton Univ. Press, Princeton, N.J., 1940.

14. G. Vidossich, A note on cardinal reflections in the category of uniform spaces, Proc. Amer. Math. Soc. 23 (1969), 53-58.

Departamento de TeOría de Funciones, FaCultad de Ciencias Matemáticas,

C/ DOCTOR MOliner 50, BurJasot, VALENCIA, SPAin 